\title{
Ultrasonography shows no abnormality of pelvic structures in women with severe idiopathic constipation
}

\author{
M A KAMM, ALISON MCLEAN, M J G FARTHING, \\ AND J E LENNARD-JONES \\ From St Marks Hospital, London and St Bartholomew's Hospital, London
}

SUmmary Patients with severe idiopathic constipation are almost exclusively women. Common presenting features include lower abdominal pain, irregular and painful menstruation, and a past history of gynaecological surgery. Pelvic ultrasonography was undertaken in 26 patients with severe idiopathic constipation and 21 age matched control women to detect any gynaecological abnormality. No difference was found in ovarian, uterine or pelvic vein morphology or size. The abdominal pain in these patients is related to the bowel disorder, and minor abnormalities of pelvic morphology on ultrasound are probably incidental findings.

Severe idiopathic constipation in patients with a normal diameter rectum and colon is almost exclusively confined to young women, ${ }^{1}$ raising the possibility that hormonal or gynaecological factors may be of aetiological significance. Further evidence comes from a questionnaire survey of 64 young women with severe idiopathic constipation in whom there was an increased prevalence of painful and irregular menstrual periods compared with an age matched control group. These patients also have an increased incidence of ovarian cystectomy and hysterectomy.'

Patients with severe idiopathic constipation nearly always experience lower abdominal pain,' but chronic lower abdominal pain is also seen in some young women not complaining of constipation. In a recent study 45 women with idiopathic lower abdominal pain and two control groups were investigated by transuterine pelvic venography. ${ }^{2}$ Women with the 'pelvic pain syndrome' showed pelvic varicosities, particularly involving the ovarian veins, and vascular stasis. The presence of dilated pelvic veins was unrelated to parity or the menstrual cycle. These dilated veins can also be seen by ultrasound scanning. ${ }^{3}$

Address for correspondence: Dr M Kamm, St Marks Hospital, City Road. London EC1V 2PS.

Accepted for publication 24 January 1989.
We have undertaken a prospective study, using ultrasound examination, in a group of severely constipated women and a matched control group of normal women, to determine whether women with severe constipation have a true increased prevalence of gynaecological disorder or any other abnormality of pelvic morphology.

\section{Methods}

PATIENTS

Twenty six women with severe idiopathic constipation (mean age 32 years, range 14-43) were studied. All patients were referred to our hospital because of severe constipation and all experienced intermittent lower abdominal pain. All the patients had a history of constipation exceeding three years and a spontaneous stated bowel frequency of less than once per week. Twenty one healthy women (mean age 33 years, range 22-47) without gastrointestinal complaints, who were hospital staff, were studied as controls.

One patient with constipation had a history of endometriosis, diagnosed at laparoscopy, and another patient had previous suspected endometriosis. Two patients had had laparotomies for abdominal pain (no cause found), and another patient had had a previous dilatation and curettage, 
ovarian cystectomy, and pelvic floor repair. None of the control group had had previous gynaecological abnormalities or surgery.

All the control subjects had regular menstrual cycles lasting 24-30 days. Twenty four of the patients had regular menstrual cycles lasting 23-30 days, and two had irregular cycles. No subject was taking the oral contraceptive. All subjects were studied in the follicular phase of the menstrual cycle, apart from six patients (four studied in the luteal phase and two had irregular menstruation) and one control (studied in the luteal phase).

Pelvic scanning was performed by a standard abdominal technique by the same observer (AMcL), with subjects having a full bladder, using a Diasonics DRF. 100L machine and $3.5 \mathrm{MHz}$ transducer. Ultrasound examination was performed systematically to examine the general morphology of the uterus, adnexa, ovaries, and pelvic veins. Ovaries were measured in three planes and the volume calculated assuming a prolate elipse shape. ${ }^{+}$Uterine dimensions were measured in the sagittal plane and the crosssectional area determined by the product of the maximum length of the uterus (from cervix to fundus) and maximum anteroposterior diameter at the fundus. ${ }^{+}$Dilated pelvic veins were defined as multiple small tubular structures in the immediate parauterine region, separate from the ovaries. These have been shown to correlate with dilated veins on pelvic venography and laparoscopy. ${ }^{3}$

Table Pelvic ultrasound findings in women with idiopathic constipation and control subjects

\begin{tabular}{|c|c|c|}
\hline & Controls & Patients \\
\hline Number studied & 21 & 26 \\
\hline Normal & 15 & 20 \\
\hline Uterine fibroids & 4 & 2 \\
\hline Polycystic ovary syndrome & 1 & 0 \\
\hline Borderline PCOS (6-9 $\mathrm{cm}^{3}$ volume bilaterally) & 0 & 3 \\
\hline \multicolumn{3}{|l|}{ Prominent parametrial veins } \\
\hline with fibroids & 1 & 1 \\
\hline without fibroids & () & () \\
\hline \multicolumn{3}{|l|}{ Thick endometrium (sagittal scan, each wall } \\
\hline$>0.5 \mathrm{~cm}$ thick $)$ & 0 & 1 \\
\hline \multicolumn{3}{|l|}{ Other findings: } \\
\hline Solitary large cyst ( $>3 \mathrm{~cm}$ diam in foll phase) & 0 & $1(5 \mathrm{~cm})$ \\
\hline Ureterocoele (unilateral) & 1 & 0 \\
\hline \multicolumn{3}{|l|}{ Measurements: (mean (SEM)) } \\
\hline Uterine size $\left(\mathrm{cm}^{2}\right)$ & $30 \cdot 7(3 \cdot 5)$ & $32 \cdot 5(3 \cdot 6)$ \\
\hline Endometrial thickness (cm) & $0.9(0 \cdot 1)$ & $0 \cdot 8(0 \cdot 1)$ \\
\hline $\mathrm{R}$ ovarian volume $\left(\mathrm{cm}^{3}\right)$ & $4 \cdot 5(0 \cdot 6)$ & $4 \cdot 1(0 \cdot 6)$ \\
\hline L ovarian volume $\left(\mathrm{cm}^{3}\right)$ & $4 \cdot 2(1.9)$ & $5.7(1.9)$ \\
\hline
\end{tabular}

One control and one patient had fibroids and dilated parametrial veins. The patient with a solitary large ovarian cyst had previous endometriosis. There was no significant difference $(p>0 \cdot(05)$ between the patients and control subjects in relation to uterine size, endometrial thickness, or ovarian volume (Student's $t$ test).
Polycystic ovaries were defined by the presence of either multiple cysts $(\geq 10)$ from 2 to $18 \mathrm{~mm}$ in diameter distributed evenly around the ovarian periphery with an increased amount of stroma, or multiple small cysts $2-4 \mathrm{~mm}$ in diameter distributed throughout the abundant stroma. Ovarian volume was also required to be increased $\left(>9 \mathrm{~cm}^{3}\right)$ to permit a diagnosis of polycystic ovary. ${ }^{+}$Ovarian volumes of $6-9 \mathrm{~cm}^{3}$ were regarded as above the normal range and possibly polycystic.

\section{Results (Table)}

There was no difference in the prevalence of ovarian or uterine abnormality between the control and patient group. In addition there was no dilatation of the pelvic veins in the constipated women.

\section{Discussion}

Using ultrasound scanning this study has found no structural pelvic abnormality in constipated women. There was no abnormality in ovarian, pelvic vein, or uterine morphology or size in the constipated group compared with a group of normal women.

In contrast with a previous study, ' most of the patients in this study experienced regular menstrual cycles. This difference may relate to the sampling of a different subpopulation of patients, or more likely, relates to the difference in the response obtained by a questionnaire' and direct personal interview. In both the previous' and the present study there was a higher incidence of previous gynaecological surgery in the patient group compared with a healthy control group of women.

We believe it is likely that a lack of appreciation of the true incidence of ovarian cysts in the normal population leads to the false assumption in some women with constipation that the presence of small cysts is abnormal and related to their abdominal pain . A high incidence of polycystic ovaries has recently been described in normal women." In that study pelvic ultrasound scanning was carried out in 257 normal volunteers who had not sought treatment for menstrual disturbances, infertility, or hirsutism. Twenty two per cent had polycystic ovaries and there was a high correlation between this ultrasound finding and an irregular menstrual history.

We paid particular attention to the pelvic veins in this study - the absence of venous dilatation suggests that the constipated women are suffering from a different disorder and have a different actiology for their pain compared with women with idiopathic chronic lower abdominal pain. ${ }^{2}$

The association of chronic lower abdominal pain and severe constipation requires greater recognition. 
Ovarian and other pelvic abnormalities discovered on ultrasound scanning are likely to be incidental.

MAK was supported by the St Mark's Research Foundation.

\section{References}

1 Preston DM, Lennard-Jones JE. Severe chronic constipation of young women: idiopathic slow transit constipation'. Gut 1986; 27: 41-8.

2 Bcard RW, Pearce S, Highman JH, Reginald PW. Diagnosis of pelvic varicosities in women with chronic pelvic pain. Lancet 1984; ii: 946-9.
3 Adams J, Beard RW, Franks S, Pearce S, Reginald PW, Sutherland IA. Pelvic ultrasound findings in women with chronic pelvic pain: correlation with laparoscopy and venography. 24th British Congress of Obstetrics and Gynaecology, Cardiff. 1986. [Abstract no 80).

4 Adams J, Franks S, Polson DW, et al. Multifollicular ovaries: clinical and endocrine features and response to pulsatile gonadotrophin releasing hormone. Lancet 198.5: ii: $1375-9$.

5 Sample WF, Lippe BM. Gyepes MT. Gray-scale ultrasonography of the normal female pelvis. Radiology 1977; 125: 477-83.

6 Polson DW, Wadsworth J, Adams J, Franks S. Polycystic ovaries - a common finding in normal women. Lancet 1988: i: 87()-2. 